\title{
Size isn't all that matters: noticing differences in size and temporal order
}

\author{
Elaine B. Wencil ${ }^{1,2}{ }^{*}$, Petya Radoeva ${ }^{3}$ and Anjan Chatterjee ${ }^{1,2,4}$ \\ 1 Center for Cognitive Neuroscience, University of Pennsylvania, Philadelphia, PA, USA \\ 2 Center for Functional Neuroimaging, University of Pennsylvania, Philadelphia, PA, USA \\ ${ }^{3}$ Department of Neuroscience, University of Pennsylvania, Philadelphia, PA, USA \\ ${ }^{4}$ Department of Neurology, University of Pennsylvania, Philadelphia, PA, USA
}

Edited by:

Anna C. Nobre, University of

Oxford, UK

Reviewed by:

Jennifer T. Coull, Universite

AixMarseille 1, France

Anne Kühn, Max Planck Institute for

Neurological Research, Germany

*Correspondence:

Elaine B. Wencil, Department of

Neurology, 3 West Gates, 3400 Spruce

St., Philadelphia, PA 19104, USA.

e-mail:ewencil@gmail.com
The ability to represent time and size is essential for thought and action. These domains have traditionally been investigated independently. However, the processing of events in time and space is postulated to have considerable anatomical and behavioral overlap. Here we formally tested for associations and dissociations of abilities in these domains. We examined patterns of impairments in temporal order and relative size judgments in 40 patients with unilateral brain lesions and 20 age-matched control participants. While brain damage can impair both size and temporal order judgments (TOJ), we did not find evidence for global hemispheric differences. When patients were analyzed individually compared to control subjects, we found double dissociations in performances on both kinds of judgments. Voxel lesion symptom mapping allowed us to investigate shared and unique contributions of brain damage to deficits in judgments noticing differences in temporal order and in spatial extent. We found that size and temporal order estimations have overlapping cortical vulnerabilities within the left inferior frontal gyrus, left superior temporal cortex, and bilateral inferior parietal lobule. However, vulnerability unique toTOJ occurred with damage predominantly in left lateralized regions involving inferior and middle frontal cortex and inferior parietal lobule. Conversely, vulnerability unique to size judgments occurred with damage predominantly in right lateralized regions in the supramarginal gyrus and superior temporal cortex. These data provide evidence for interactions between the processing of spatial extent and temporal order; however, they do not provide evidence for right lateralized systems. Keywords: temporal order judgment, temporal perception, size perception, inferior parietal lobule, voxel lesion symptom
mapping

\section{INTRODUCTION}

Perceiving spatial and temporal dynamics arguably underlies all thought and actions (Kant, 1929; Fraisse, 1963). Although the temporal dimension is often overlooked, precise representation of how events unfold in time is necessary for such diverse activities as speech perception, object identification and motor coordination. While many aspects of spatial processing have been extensively examined, how the cortex processes temporal information, such as determining the duration of intervals (Rao et al., 2001; Coull et al., 2008) or sequencing of events remains unclear (for review Battelli et al., 2008).

A large body of research arising from the animal literature describes the flow of visual spatial information from primary visual cortex (V1) along the dorsal or "where" pathway to the parietal cortex (Mishkin and Ungerleider, 1983; Goodale and Milner, 1992). Size, a variable of spatial extension, may be represented, in parallel, by both the dorsal and ventral ("what") streams. Behavioral double dissociations in patient studies and anatomical double dissociations in neuroimaging studies implicate the intraparietal sulcus within the dorsal stream, when computing size for grasping and the lateral occipital cortex of the ventral stream when perceiving size for object discrimination (Goodale et al., 1991; Faillenot et al., 1997; Cavina-Pratesi et al., 2007).
In comparison to spatial perception, relatively little is understood about the neural basis of temporal perception. Internal clock models are commonly invoked to explain processing of sub-second intervals (Treisman, 1963; Gibbon, 1977). Though it remains unclear how brain regions map onto an internal clock, cortical regions such as inferior parietal cortex, superior temporal cortex, supplementary motor area (SMA) and dorsal-lateral prefrontal cortex are frequently implicated in processing temporal information such as intervals (Onoe et al., 2001; Rao et al., 2001; Lewis and Miall, 2003a; Wencil et al., 2010), temporal order (Battelli et al., 2007) and temporal orienting (Coull and Nobre, 1998; Nobre et al., 2007). Recently, based largely on temporal order and motion literature, right posterior superior temporal cortex and temporalparietal junction (TPJ) have been hypothesized to serve as part of a "when" pathway (Battelli et al., 2007) thought to be distinct from yet highly interactive with the adjacent "what" and "where" pathways. However, others have emphasized left posterior parietal cortex involvement in sequencing of events and temporal orienting (Coull and Nobre, 1998; Coull, 2004; Davis et al., 2009).

Functional overlap of encoding spatial and temporal extent supports the interaction of space and time processing broadly defined. Neural representations of durations have been linked to spatially selective cells; specifically, spatially tuned lateral intraparietal (LIP) 
cells of monkeys also encode elapsed durations (Leon and Shadlen, 2003) and millisecond duration judgments can be spatially localized (Burr and Morrone, 2006; Johnston et al., 2006). Direct behavioral evidence for the integration of space and time is provided by the finding that saccadic eye movements cause compression of both duration and space (Morrone et al., 2005). Psychophysical studies also suggest that size affects time estimation. For example, larger stimuli are judged to last longer (Xuan et al., 2007) and spatial scale influences elapsed duration (DeLong, 1981; Mitchell and Davis, 1987). Spatial terms are commonly used to describe time (Casasanto and Boroditsky, 2008).

Evidence that the "what" and "where" pathways might link with the putative "when" pathway is provided by behavioral deficits of patients with right cortical lesions. Patients with right parietal damage show co-occurring spatial and temporal attention deficits (Critchley, 1953; Rorden et al., 1997; Shapiro et al., 2002). Furthermore, impairments in temporal order can be ameliorated by spatial separation (Snyder and Chatterjee, 2004). Patients with attentional disorders such as neglect show impairments not only in spatial attention, but in size (Milner and Harvey, 1995; Milner et al., 1998; Ferber and Karnath, 2001), temporal perception (Danckert et al., 2007) and temporal order (reviewed in Becchio and Bertone, 2006; Danckert et al., 2007). Furthermore, impairments of neglect patients of orienting in time are more severe than impairments seen in patients with similar cortical damage without neglect (Husain et al., 1997).

In the current study we use voxel lesion symptom mapping (VLSM) to determine the shared and unique contributions of brain damage to judgments in time and space. We assess temporal order, one aspect of temporal processing by performance on a temporal order judgment (TOJ) task with stimulus-onset-asynchronies (SOA) ranging from $40-80 \mathrm{~ms}$. This time scale, referred to as automatic timing (Lewis and Miall, 2003b), is crucial in determining the sequencing of objects in the world and to control motor behavior (Battelli et al., 2007). TOJ tasks are widely used and performance is altered by right hemisphere dysfunction (Rorden et al., 1997; Robertson et al., 1998; Snyder and Chatterjee, 2004; Woo et al., 2009); to date detailed lesion mapping has not been performed. We will compare TOJ performance with performance on a spatial task well matched for visual perception and motor response demands. Specifically, we assess spatial processing with a size judgment task where the area of two circles differs between 5-13\%. Recently, TOJ performance was shown to activate temporal-parietal junction more than a shape task (Davis et al., 2009). We attempt to strengthen these fMRI findings by using VLSM. Convergent methodologies are necessary in the cognitive neurosciences to compensate for different strengths and weaknesses of each method (Devinsky and D'Esposito, 2004; Farah and Wolpe, 2004; Rorden and Karnath, 2004). Functional imaging studies have grown in popularity and impact (Chatterjee, 2005; Fellows et al., 2005) yet lesion studies remain an important compliment to the correlation nature of functional imaging results. Compared to functional imaging, lesion studies provide stronger evidence that the localized brain area is necessary for the underlying cognitive processes. By using VLSM over traditional lesion techniques we increase the specificity of neuroanatomical claims without the pitfalls of ignoring variability in participant's performance.

\section{MATERIALS AND METHODS PARTICIPANTS}

Forty adults with chronic unilateral lesions (20 with left involvement and 20 with right involvement) were recruited from the Focal Lesion Database (Center for Cognitive Neuroscience, University of Pennsylvania). One participant was removed from analysis due to concomitant psychiatric disturbances not identified at screening. Participants were not selected based on their lesion locations or patterns of cognitive impairment. They ranged in age from 32 to 81 -years old with a mean age of 57-years old; 24 were female. For further demographic information see Table 1; for basic neuropsychological performance see Table 2. The left and right hemisphere patient populations did not differ by age $(\mathrm{t}(37)=-0.851, p=0.4)$, chronicity $(\mathrm{t}(37)=-0.07, p=0.945)$ nor size of lesion ( $\mathrm{t}(37)=0.147, p=0.159)$. Twenty matched neurologically intact controls (average age 60-years old, 14 females) were also recruited. All control participants were right-handed, native English speakers without history of neurological or psychiatric symptoms. All participants and neurologically intact controls provided written, informed consent in accordance with the policies of the University of Pennsylvania's Institutional Review Board.

\section{BEHAVIORAL TASKS}

The experiment was conducted with e-prime (Psychology Software Tools) on a $40 \mathrm{~cm}$ monitor positioned approximately $57 \mathrm{~cm}$ from the participant. The stimulus display consisted of a light-gray background with a black central fixation asterisk. The visual targets consisted of two black circles measuring $1.0^{\circ}$ in height and in width (see Figure 1). One circle contained an " $X$ " while the other contained a "+". Participants performed a minimum of 16 trials of a target detection task to get familiar with the stimulus set and response mapping. Following training, participants performed eight blocks of a size judgment task and eight blocks of a temporal order judgment task. Each block contained 16 trials. Blocks and trials were randomized. Participants responded by keypress with their ipsilesional hand. Control participants were counterbalanced to left or right-handed responses.

\section{TARGET DETECTION TRAINING TASK}

Participants performed a target detection task to gain familiarity with the stimuli set and comfort with the response mapping. Each training trial began with a central asterisk that participants were instructed to fixate on for the duration of the trial. After $2000 \mathrm{~ms}$, the target circle appeared with equal probabilities in one of the four quadrants (i.e., top left, top right, bottom left or bottom right) of the display. The target circle, containing either an " $x$ " or a "+", was presented for $500 \mathrm{~ms}$. Following target offset, subjects were given $3500 \mathrm{~ms}$ to indicate which circle was presented (either with the internal " $x$ " or internal "+"). The participants repeated blocks of 16 trials of this training task until they reached a criterion of $80 \%$ accuracy. Once this criterion was reached they began the experimental conditions.

\section{SIZE JUDGMENT TASK}

Each trial began with a central asterisk that participants were instructed to fixate on for the duration of the trial. After $2000 \mathrm{~ms}$, the visual targets appeared in one of four arrangements: (a) two 
Table 1 | Participant demographic data.

\begin{tabular}{|c|c|c|c|c|c|c|c|}
\hline Participant & Gender & Age & Hemisphere & Location & Chronicity & \# Lesioned voxels & Cause \\
\hline MB_101 & $\mathrm{F}$ & 51 & $\mathrm{R}$ & $\mathrm{T}$ & 30 years & 3952 & Stroke \\
\hline HQ_337 & M & 80 & L & $\mathrm{P}$ & 4 years & 6111 & Stroke \\
\hline DX_444 & $\mathrm{F}$ & 74 & R & PT & 3 years & 1844 & Stroke \\
\hline KN_313 & M & 52 & $R$ & $T$ & 10 years & 2345 & Surgery for aneurysm \\
\hline TL_553 & M & 39 & $R$ & $T$ & 1 year & 6525 & Tumor resection \\
\hline KE_205 & $\mathrm{F}$ & 77 & R & $\mathrm{F}$ & 7 years & 4228 & Stroke \\
\hline MN_93 & $\mathrm{F}$ & 70 & L & $\mathrm{T}$ & 6 years & 7980 & Hemorrhage \\
\hline MF_560 & M & 58 & $\mathrm{R}$ & FP & 2.5 years & 428 & Stroke \\
\hline SR_489 & M & 70 & L & $\mathrm{T}$ & 9 years & 121 & Radiation necrosis \\
\hline LM_292 & M & 59 & L & TBG & 5 years & 37838 & Stroke \\
\hline CC_517 & $\mathrm{F}$ & 57 & L & $\mathrm{F}$ & 5 years & 5641 & Stroke \\
\hline KX_570 & M & 54 & $R$ & $\mathrm{P}$ & 4 months & 1783 & Hematoma \\
\hline CD_141 & $\mathrm{F}$ & 46 & L & $\mathrm{T}$ & 5 years & 1068 & Stroke \\
\hline FC_83 & M & 64 & $R$ & FTP & 8 years & 8040 & Stroke \\
\hline SL_41 & M & 63 & L & PT & 7 years & 37402 & Stroke \\
\hline UM_103 & M & 49 & L & $B G$ & 6 years & 22093 & Stroke \\
\hline TD_440 & $\mathrm{F}$ & 55 & $R$ & $\mathrm{~F}$ & 6 years & 3945 & Tumor resection \\
\hline NF_113 & $\mathrm{F}$ & 55 & $R$ & $\mathrm{~F}$ & 12 years & 7158 & Stroke \\
\hline TM_544 & $\mathrm{F}$ & 40 & $\mathrm{R}$ & FP & 3 years & 9092 & Aneurysm \\
\hline NQ_87 & $\mathrm{F}$ & 65 & $R$ & $\mathrm{~F}$ & 8 years & 1020 & Stroke \\
\hline CN_541 & M & 41 & L & $\mathrm{F}$ & 2.5 years & 1348 & Tumor resection \\
\hline NE_567 & $\mathrm{F}$ & 40 & L & $\mathrm{F}$ & 11 years & 11180 & Stroke \\
\hline QN_573 & $\mathrm{F}$ & 43 & L & FP & 13 years & 1719 & Stroke \\
\hline NC_486 & $\mathrm{F}$ & 54 & $R$ & PT & 5 years & 2784 & Stroke \\
\hline DS_564 & $\mathrm{F}$ & 73 & $R$ & FP & 5 years & 578 & Stroke \\
\hline
\end{tabular}

T, temporal; P, parietal; F, frontal; BG, basal ganglia, ACC, anterior cingulate cortex.

circles with $7^{\circ}$ of horizontal separation $3.5^{\circ}$ above the horizontal axis; (b) two circles with $7^{\circ}$ of horizontal separation $3.5^{\circ}$ below the horizontal axis; (c) two circles with $7^{\circ}$ of vertical separation $3.5^{\circ}$ left of the vertical axis and (d) two circles with $7^{\circ}$ of vertical separation $3.5^{\circ}$ right of the vertical axis. In each trial, one circle was the standard diameter $\left(1^{\circ}\right)$ while the other circle was either $5,8,10$, or $13 \%$ larger in area. The visual targets were presented for $500 \mathrm{~ms}$. Following target offset, subjects were given $3500 \mathrm{~ms}$ to indicate whether the circle with the internal "+" or the circle with the internal " $X$ " was larger. All trials were counterbalanced for target location and whether the circle with " + " or " $X$ ” was the standard size. The diameter increments were chosen based on pilot testing so that there was a scaled level of difficulty that ranged from $90 \%$ accuracy with the largest size asynchrony to $75 \%$ accuracy in smallest size asynchrony.

\section{TEMPORAL ORDER JUDGMENT TASK}

Similarly to the size judgment task, each trial began with a $2000 \mathrm{~ms}$ fixation asterisk. Following fixation the target circles appeared in the same spatial configuration as in the size judgment task. The TOJ task differed in that the second circles had a delayed onset of $40,53,67$, or $80 \mathrm{~ms}$ following the target circle. The visual targets then remained on the screen for a total of $500 \mathrm{~ms}$. Participants were given $3500 \mathrm{~ms}$ to indicate whether the circle with the internal 
Table 2 | Participant neuropsychological performance.

\begin{tabular}{|c|c|c|c|c|c|}
\hline \multirow[b]{2}{*}{ Participant } & \multirow[b]{2}{*}{ Hemisphere } & \multicolumn{2}{|c|}{ Verbal } & \multicolumn{2}{|c|}{ Visuo-spatial } \\
\hline & & $\begin{array}{l}\text { WAIS- } \\
\text { Info }\end{array}$ & AMNART & $\begin{array}{l}\text { Line } \\
\text { cancellation }\end{array}$ & $\begin{array}{l}\text { Line } \\
\text { bisection }\end{array}$ \\
\hline MB_101 & $\mathrm{R}$ & 20 & 121 & na & -9 \\
\hline HQ_337 & $\mathrm{L}$ & 10 & na & 39 & -10.5 \\
\hline KT_325 & $\mathrm{R}$ & na & na & 39 & -5 \\
\hline DX_444 & $\mathrm{R}$ & na & 99 & 40 & -4 \\
\hline KN_313 & $\mathrm{R}$ & 17 & 118 & 40 & 6 \\
\hline MK_428 & $\mathrm{L}$ & na & na & 40 & 2.5 \\
\hline XD_3 & $\mathrm{L}$ & na & na & na & na \\
\hline TL_553 & $\mathrm{R}$ & 22 & 121 & na & na \\
\hline KE_205 & $\mathrm{R}$ & 23 & 115 & 40 & -1 \\
\hline MN_93 & $\mathrm{L}$ & 11 & 102 & 40 & -8 \\
\hline MF_560 & $\mathrm{R}$ & 10 & 97 & 40 & na \\
\hline SR_489 & $\mathrm{L}$ & 20 & 118 & 40 & na \\
\hline DF_552 & $\mathrm{R}$ & na & 106 & 40 & na \\
\hline NW_561 & $\mathrm{L}$ & 21 & 111 & 40 & na \\
\hline OM_559 & $L$ & na & na & & na \\
\hline DF_316 & $\mathrm{R}$ & na & na & 40 & 6 \\
\hline LM_292 & $\mathrm{L}$ & na & na & 40 & 1 \\
\hline CC_517 & $L$ & 14 & 107 & 39 & na \\
\hline KX_570 & $\mathrm{R}$ & 18 & 103 & 40 & na \\
\hline CD_141 & $\mathrm{L}$ & 11 & 113 & 40 & -4 \\
\hline FC_83 & $\mathrm{R}$ & 26 & 114 & 40 & -4 \\
\hline SL_41 & $\mathrm{L}$ & na & na & 40 & -12 \\
\hline UM_103 & $L$ & 10 & na & 40 & 0 \\
\hline TD_440 & $\mathrm{R}$ & na & na & 40 & 0 \\
\hline NF_113 & $\mathrm{R}$ & na & 123 & 40 & -1 \\
\hline TM_544 & $\mathrm{R}$ & na & na & na & na \\
\hline BE_249 & $\mathrm{L}$ & 18 & 105 & 40 & na \\
\hline DU_532 & $L$ & 9 & 106 & 40 & na \\
\hline CP_430 & $\mathrm{L}$ & na & na & 40 & -13.5 \\
\hline KG_215 & $L$ & 14 & 106 & 40 & -1 \\
\hline GU_412 & $\mathrm{L}$ & 7 & na & 40 & 6 \\
\hline KX_481 & $\mathrm{R}$ & na & na & 40 & 3 \\
\hline NS_569 & $\mathrm{R}$ & 24 & 125 & 40 & na \\
\hline NQ_87 & $\mathrm{R}$ & 16 & 113 & na & -0.15 \\
\hline CN_541 & $\mathrm{L}$ & 23 & na & 40 & na \\
\hline NE_567 & $\mathrm{L}$ & 18 & 115 & 40 & na \\
\hline QN_573 & $L$ & na & na & 40 & na \\
\hline NC_486 & $\mathrm{R}$ & na & na & na & na \\
\hline DS_564 & $\mathrm{R}$ & na & na & na & na \\
\hline
\end{tabular}

AMNART (American National Adult Reading Test) score is an estimated verbal IO with a mean of $100 \pm 15$. Line Cancellation data are the number of lines cancelled with a max of 40. Line bisection is in $\mathrm{mm}$ with - corresponding to leftward deviations. na indicates not administered. WAIS-III Information subsection, data are the number correct with a max score of 28.

"+" or the circle with the internal " $x$ " appeared first. The temporal offsets were chosen based on pilot testing so that there was a scaled level of difficulty that ranged from $90 \%$ accuracy with the longest temporal asynchrony to $75 \%$ accuracy in briefest temporal asynchrony.



VOXEL LESION SYMPTOM MAPPING

Clinical CT or MRI brain scans of each participant were provided through the Focal Lesion Database. Lesion masks for each participant were drawn by a senior neurologist blind to patient's performance using MRIcro software (Rorden and Brett, 2000). Lesions were drawn on $2 \mathrm{~mm} \times 2 \mathrm{~mm} \times 2 \mathrm{~mm}$ MNI templates tilted in the same axial planes of the source images. Each template was then realigned to a common axial angle. We engaged VLSM, a form of statistical parametric mapping, to map behavioral performance to brain damage on a voxel-by-voxel basis (Kimberg et al., 2007).

Statistical analysis was conducted with Voxbo image-analysis software (www.voxbo.org). In contrast with BOLD analysis, where the dependent value is the signal value in a given voxel and behavior the independent value, in VLSM the presence or absence of a lesion in each voxel represents the independent variable and behavioral performance is the dependent variable. In the current study, we regressed $z$-scores for TOJ performance and $z$-scores for size discrimination performance on lesion status scores across participants. To assess statistical significance, we conducted non-parametric permutation tests where 1000 randomly generated permutations of the lesion status mapped to the $z$-score serve as a null distribution of the data. A maximum statistic across the brain is calculated for each permutation and thresholds for significance calculated from 
the 95th percentile of this distribution to ensure a family-wise false positive rate of 0.05 . Thus, using this maximum statistic corrects for multiple comparisons, without the assumptions that underlie parametric statistics (Nichols and Holmes, 2002).

Voxel lesion symptom mapping analysis, in accordance with Bates et al. (2003) seminal paper is conducted with all participants, both those with and without deficits. By incorporating the lesions of both "normal" and "deficit" participants and by using their performance as a continuous rather than a categorical variable, VLSM overcomes limits of traditional lesion analyses. By including lesions of participants with normal performances, VLSM increases specificity. For example, if every patient with a deficit had left angular gyrus lesions, but every patient without a deficit also had left angular gyrus lesions, traditional analyses would erroneously conclude that left angular gyrus lesions was causally related to the deficit. In addition, unlike traditional lesion analysis, consideration can be given to parametric variation in performance which means that one does not have to adopt conventional thresholds (such as 2 SD), create dichotomous groups and then ignore variability within these groups. For further details of this logic, see Kimberg et al. (2007) and for recent applications see Wu et al. (2007) and Amorapanth et al. (2009).

Given that VLSM results differ in dependence of used parameters it is important to consider factors such as lesion size in VLSM analysis (Kimberg et al., 2007). In our population there was no correlation between performance on TOJ or size judgment with either chronicity (TOJ: $r=-0.221, p=0.18$; size: $r=-0.055, p=0.74$ ) or lesion size determined by number of lesion voxels (TOJ: $r=0.09$, $p=0.59$; size: $r==0.026, p=0.88$ ). Therefore we did not include either of these as factors in our VLSM.

\section{RESULTS}

\section{BEHAVIORAL RESULTS}

\section{Group analyses}

The 20 matched controls performed well on both the size discrimination and TOJ tasks (see Figures 2A,B). As expected, performance scaled with increased size and increased temporal asynchrony
$(F(3,17)=65.41, p<0.001)$. However, performance also varied by task $(F(1,19)=15.362, p<0.01)$ where control participants were less accurate on the TOJ task $(80 \%)$ than the size discrimination task $(88 \%)$. Performance across both tasks correlated significantly $(r=0.46, p<0.05)$ (see Figure 3A).



B

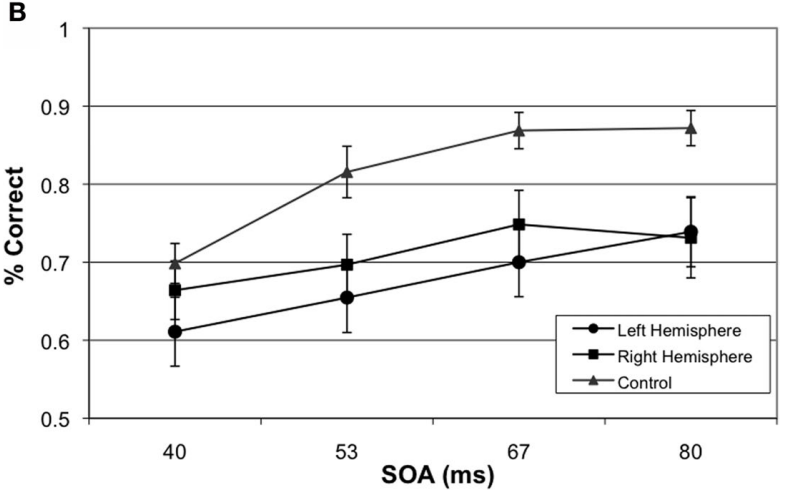

FIGURE 2 | Group-wise Behavioral Results. (A) Performance of left hemisphere involvement, right hemisphere involvement and age-matched participants on size judgment task and (B) Performance of left hemisphere involvement, right hemisphere involvement and age-matched participants on TOJ.
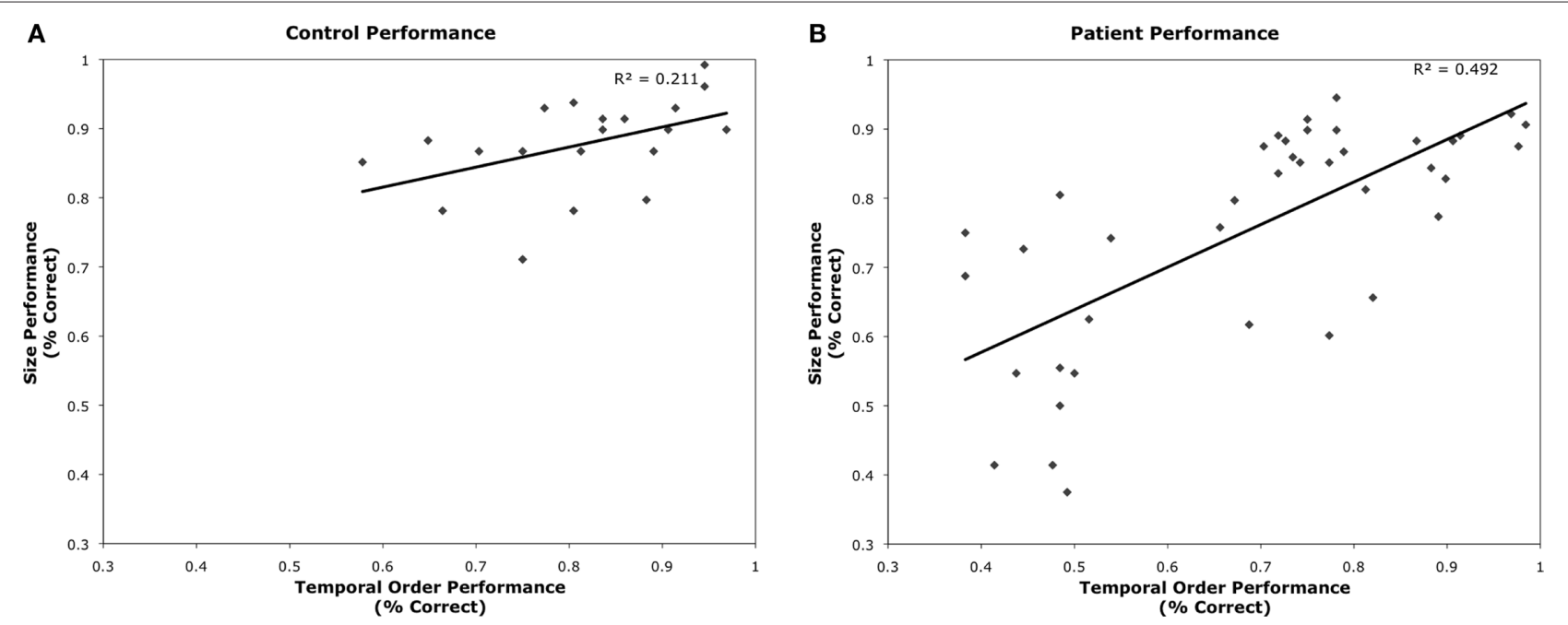

FIGURE 3 | Plots of TOJ versus Size Judgment performance for (A) age-matched controls and (B) patient participants. 
Overall participants were less accurate than controls on the TOJ and size discrimination tasks, with $70 \%$ and $76 \%$ performance respectively $(F(2,56)=5.18, p<0.01)$. Paired contrasts, with correction for multiple comparisons, revealed significant differences in performances between participants with left hemisphere involvement and controls $(p=0.018)$ as well as significant differences in performance between participants with right hemisphere involvement and controls $(p=0.027)$ collapsed across conditions. Interestingly, there was no difference on performance between participants with left versus right hemisphere involvement $(p=0.999)$.

Performance across TOJ and size judgment tasks was significantly correlated $(r=0.70, p<0.001)$ (Figure 3B). There were no significant correlations between performance on TOJ or size judgment with either chronicity (TOJ: $r=-0.221, p=0.18$; size: $r=-0.055, p=0.74)$ or lesion size determined by number of lesioned voxels (TOJ: $r=0.09, p=0.59$; size: $r=0.026, p=0.88$ ). Performance on TOJ correlated with participant's age $(r=-0.364$; $p=0.02)$; performance on size judgment showed a similar trend $(r=-0.299, p=0.06)$.

\section{Case analyses}

We analyzed each participant's performance in a case series manner. Each participant's performance was compared to the control groups' performance by repeated measures ANOVA (difficulty $\times$ group) independently for size judgment and TOJ. Results of each of these tests are presented in Table 3. When comparing a single case versus a small control group in this fashion, we accept untestable assumptions about the homogeneity of variance and covariance (Corballis, 2009; Crawford et al., 2009). Without using corrections it is difficult to make conclusions about the populations that the case and control group arise from, nonetheless, these analysis are conservative enough to allow us to make claims about the comparison of the case to the group of controls. Therefore, impairment on a task was defined by a significant main effect of group. Twenty-four of the participants had no impairments while nine of the participants had impairments across both size judgment and TOJ tasks. Critically, six participants demonstrated double dissociations in performance on size and temporal order judgments (see Figure 4). Three participants had selective TOJ impairment, as defined by significantly worse performance than controls on time judgments but not on size judgments. Conversely, three participants had selective size judgment impairment.

\section{VLSM RESULTS}

\section{Size judgment task}

Permutation analyses revealed that mapwise $t$-statistic threshold with a significance level of $p<0.05$ for size discrimination was 2.9 for left hemisphere involvement and 2.7 for right hemisphere involvement. Impairment in size discrimination correlated significantly with lesions in left posterior orbital gyrus, left inferior frontal gyrus extending across the pars opercularis and pars triangularis, left posterior middle frontal, left white matter undercutting posterior superior temporal and supramarginal gyrus, right posterior superior temporal gyrus, right posterior parietal lobule extending across the supramarginal and angular gyri and right precentral gyrus. (See Figure 5A)
Table 3 | Interaction of difficulty $\times$ group forTOJ and size judgment tasks.

\begin{tabular}{|c|c|c|c|c|c|c|}
\hline & \multicolumn{2}{|c|}{ Time condition } & \multicolumn{2}{|c|}{ Size condition } & \multirow[b]{2}{*}{ Hemisphere } & \multirow[b]{2}{*}{ Location } \\
\hline & $\boldsymbol{F}$ & $p$ & $F$ & $p$ & & \\
\hline MN_93 & 8.984 & 0.007 & 1.097 & 0.308 & $L$ & $\mathrm{~T}$ \\
\hline$L M \_292$ & 15.38 & 0.001 & 3.371 & 0.082 & $\mathrm{~L}$ & TBG \\
\hline DS_564 & 6.248 & 0.022 & 3.797 & 0.066 & $\mathrm{R}$ & FP \\
\hline$M K \_428$ & 0.004 & 0.952 & 10.161 & 0.005 & $\mathrm{~L}$ & $\mathrm{ACCF}$ \\
\hline$N C \_486$ & 0.134 & 0.718 & 15.81 & 0.001 & $\mathrm{R}$ & $\mathrm{PT}$ \\
\hline MF_560 & 1.319 & 0.265 & 14.069 & 0.001 & $\mathrm{R}$ & FP \\
\hline NW_561 & 0.396 & 0.537 & 0.235 & 0.634 & $\mathrm{~L}$ & $\mathrm{~F}$ \\
\hline CC_517 & 0.134 & 0.718 & 0.138 & 0.714 & $\mathrm{~L}$ & $\mathrm{~F}$ \\
\hline KG_215 & 0.087 & 0.771 & 0.092 & 0.764 & $\mathrm{~L}$ & $\mathrm{~F}$ \\
\hline GU_412 & 0.087 & 0.771 & 0.96 & 0.339 & $\mathrm{~L}$ & $\mathrm{~F}$ \\
\hline CN_541 & 2.198 & 0.155 & 0.001 & 0.973 & $\mathrm{~L}$ & $\mathrm{~F}$ \\
\hline NE_567 & 0.336 & 0.569 & 0.092 & 0.764 & $L$ & $\mathrm{~F}$ \\
\hline XD_3 & 0.71 & 0.41 & 0.006 & 0.938 & $\mathrm{~L}$ & $\mathrm{FT}$ \\
\hline OM_559 & 1.012 & 0.327 & 0.001 & 0.973 & $L$ & $P$ \\
\hline BE_249 & 0.237 & 0.632 & 0.006 & 0.938 & $\mathrm{~L}$ & $P$ \\
\hline SR_489 & 0.521 & 0.479 & 0.067 & 0.798 & $\mathrm{~L}$ & $\mathrm{~T}$ \\
\hline CD_141 & 0.746 & 0.398 & 0.037 & 0.85 & $L$ & $\mathrm{~T}$ \\
\hline DU_532 & 2.053 & 0.168 & 2.97 & 0.101 & $\mathrm{~L}$ & $\mathrm{~T}$ \\
\hline KE_205 & 0.595 & 0.45 & 0.504 & 0.487 & $\mathrm{R}$ & $\mathrm{F}$ \\
\hline DF_552 & 0.336 & 0.569 & 0.28 & 0.603 & $\mathrm{R}$ & $\mathrm{F}$ \\
\hline TD_440 & $<0.001$ & 0.992 & 0.874 & 0.362 & $\mathrm{R}$ & $\mathrm{F}$ \\
\hline NQ_87 & 0.835 & 0.372 & 0.037 & 0.85 & $\mathrm{R}$ & $\mathrm{F}$ \\
\hline NF_113 & 0.423 & 0.523 & 0.138 & 0.714 & $\mathrm{R}$ & $\mathrm{F}$ \\
\hline NS_569 & 0.491 & 0.492 & 2.244 & 0.151 & $\mathrm{R}$ & FTP BG \\
\hline TM_544 & 0.05 & 0.825 & 0.021 & 0.885 & $\mathrm{R}$ & $\mathrm{FP}$ \\
\hline KX_570 & 1.992 & 0.174 & 0.412 & 0.529 & $\mathrm{R}$ & $P$ \\
\hline KX_481 & 0.629 & 0.438 & 0.006 & 0.938 & $\mathrm{R}$ & $P$ \\
\hline MB_101 & 1.666 & 0.212 & 1.346 & 0.26 & $\mathrm{R}$ & $\mathrm{T}$ \\
\hline KN_313 & 0.746 & 0.398 & 0.356 & 0.558 & $\mathrm{R}$ & $\mathrm{T}$ \\
\hline TL_553 & 2.414 & 0.137 & 0.174 & 0.682 & $\mathrm{R}$ & $\mathrm{T}$ \\
\hline UM_103 & 13.23 & 0.002 & 44.616 & $<0.001$ & $L$ & $B G$ \\
\hline CP_430 & 11.723 & 0.003 & 22.702 & $<0.001$ & $\mathrm{~L}$ & $F$ \\
\hline HQ_337 & 7.359 & 0.014 & 13.237 & 0.002 & $L$ & $P$ \\
\hline$S L \_41$ & 8.562 & 0.009 & 52.457 & $<0.001$ & $\mathrm{~L}$ & PT \\
\hline QN_573 & 11.241 & 0.003 & 4.726 & 0.043 & $L$ & FP \\
\hline KT_325 & 8.984 & 0.007 & 21.641 & $<0.001$ & $\mathrm{R}$ & $\mathrm{FT}$ \\
\hline FC_83 & 8.562 & 0.009 & 29.599 & $<0.001$ & $\mathrm{R}$ & FTP \\
\hline VQ_230 & 9.415 & 0.006 & 44.616 & $<0.001$ & $\mathrm{R}$ & $P$ \\
\hline DF_316 & 8.151 & 0.01 & 22.702 & $<0.001$ & $\mathrm{R}$ & $P$ \\
\hline
\end{tabular}

Since performance on size and TOJ were correlated, some variance in performance on each of these tasks could be accounted for by deficits on the complementary task. Therefore, an additional VLSM performed on the residuals was conducted to formally test for correlations between damaged brain regions and impaired performance on size discrimination independent of deficits on temporal order discrimination (Amorapanth et al., 2009). We regressed size discrimination impairment on temporal order discrimination impairment and derived residuals were used for further analysis. Permutation analyses on the resulting 
A

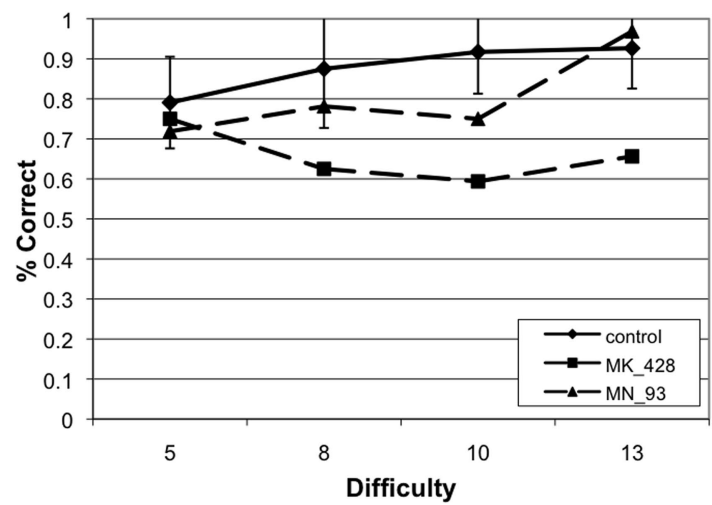

B

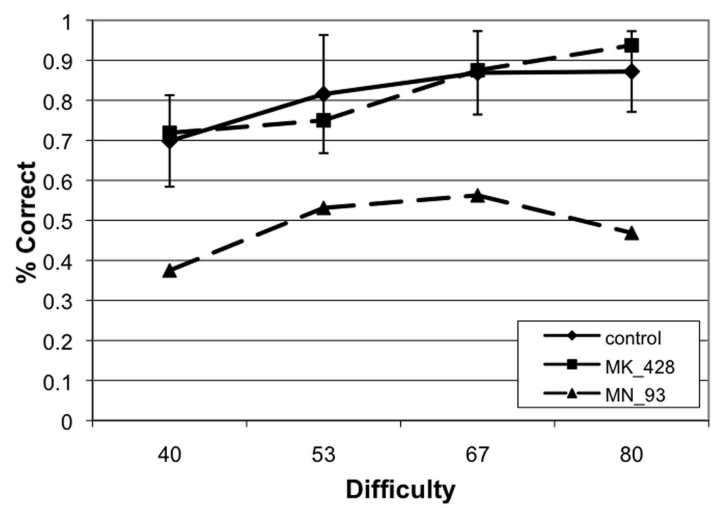

FIGURE 4 | Example of two cases that provide evidence of a double dissociation between performance on (A) size judgment and (B) TOJ performance.

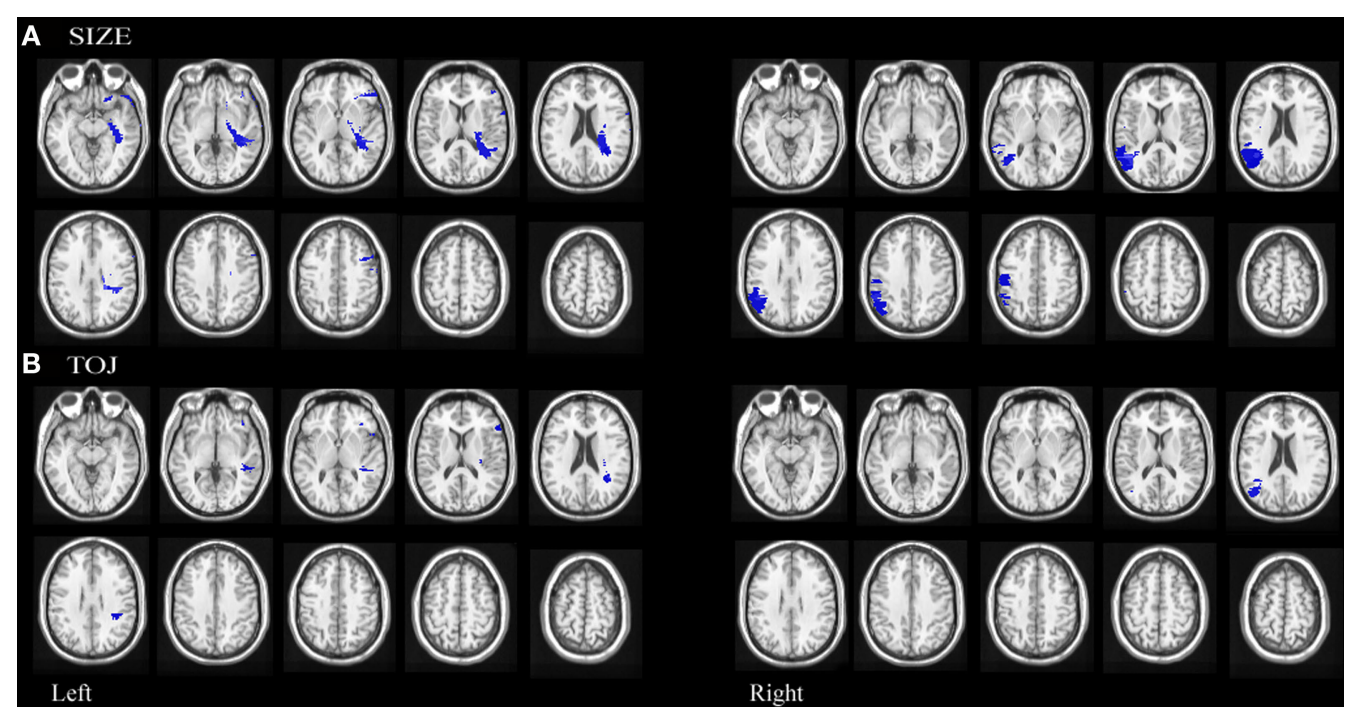

FIGURE 5 | Statistical maps of neuroanatomic regions in which damage correlated significantly (using permutation statistics) with (A) impairment on size discrimination and (B) temporal order discrimination.

residuals revealed that mapwise $t$-statistic threshold with a significance level of $p<0.05$ was 3.0 for left hemisphere involvement and 3.1 for right hemisphere involvement. Impairment on size discrimination, independent of performance on temporal order discrimination, correlated significantly with lesions in right posterior superior temporal and right supramarginal gyrus. (See Figure 6A)

\section{Temporal order judgment task}

Permutation analyses revealed that mapwise $t$-statistic threshold with a significance level of $p<0.05$ for temporal discrimination was 3.6 for left hemisphere involvement and 3.3 for right hemisphere involvement. Impairment in TOJ discrimination correlated significantly with lesions in left inferior frontal pars opercularis, left white matter undercutting the posterior superior temporal gyrus, left white matter undercutting the supramarginal gyri and right angular gyrus. (see Figure 5B)
We also performed a VLSM analysis on the residuals to formally test for correlations between damaged brain regions and impaired performance on temporal order discrimination independent of performance on size discrimination. Permutation analyses on the resulting residuals revealed that mapwise $t$-statistic threshold with a significance level of $p<0.05$ was 2.6 for left hemisphere involvement and 2.1 for right hemisphere involvement. Impairment on temporal order discrimination, independent of deficit on size discrimination, correlated significantly with lesions in left inferior frontal and posterior middle frontal gyri, left posterior parietal gyrus extending across the supramarginal and angular gyri, left pre central gyrus as well as right supramarginal gyrus. (See Figure 6B)

\section{DISCUSSION}

Regions of the inferior parietal cortex have previously been implicated in the processing of objects across space and time (Husain et al., 1997; Battelli et al., 2001, 2003; Davis et al., 2009). In the 




FIGURE 6 | Statistical maps of neuroanatomic regions in which damage correlated significantly (using permutation statistics) with (A) residuals resulting from size performance regressed againstTOJ performance, representing regions where lesions correlate with variance associated with size performance independent of TOJ performance and (B) residuals resulting from TOJ performance regressed against size performance representing regions where lesions correlate with variance associated with TOJ performance independent of size performance. current study we found double dissociations in performances on TOJ and size judgments tasks. This argues that even though previous studies have shown TOJ and size rely on similar cortical regions and have been shown to influence each other, they are not completely overlapping processing systems. Although we found that size and time do have overlapping cortical vulnerabilities, processing unique to time judgments was impaired predominantly by damage to left lateralized regions. Conversely, with the caveat of reduced power to detect left parietal involvement, processing unique to size judgments seems to be impaired predominantly by damage to right lateralized regions. Importantly, we are not arguing against right-sided cortical involvement in TOJ tasks or against left-sided cortical involvement in size judgments as evidenced by bilateral posterior parietal found in both main effect and residual VLSM analysis. We are arguing that the mechanisms involved uniquely in temporal resolution are predominantly left lateralized whereas those involved in spatial resolution are right lateralized. As the first systematic lesion mapping of TOJ, we expand on the previous findings to show that in addition to previously identified right parietal regions, left parietal and frontal regions also function as part of the "when pathway". This finding helps bridge the previous lesion data with the recent fMRI finding of left temporal-parietal junction activation during TOJ (Davis et al., 2009).

\section{BEHAVIORAL RESULTS SUPPORT PARTIALLY INDEPENDENT PROCESSING STREAMS}

The forms of the stimuli were similar across conditions; only the domain (size or temporal order) differed. Given the very similar demands across the two tasks, it is not surprising that performance across tasks were highly correlated. However, double dissociations were found. Three patients displayed a selective TOJ deficit and conversely three patients displayed a selective size judgment deficit.
Although a significant correlation between performances on the two tasks suggests that size and TOJ processing are interrelated as others have shown (Danckert et al., 2007), our data makes the possibility of a unified, completely overlapping spatial and temporal estimators unlikely. There is, at least, partial independence of the processing streams.

\section{OVERLAPPING CORTICAL REPRESENTATIONS}

Lesions of left inferior frontal cortex correlated with impaired performance on both the size judgment and TOJ tasks. This region is consistent with a reported anterior magnitude system localized to left prefrontal cortex involved with complex calculations and planning (Walsh, 2003). We are not claiming that TOJ is a simple magnitude task but that both the size judgment and TOJ tasks share many common cognitive processes, including manipulation of magnitude information, that relies upon left inferior frontal cortex. Damage to the white matter underlying the left posterior superior temporal cortex was correlated with impaired performance on both the size judgment and TOJ tasks. Posterior temporal cortex is part of the classical ventral system that is involved in processing for perception (Goodale et al., 1991). Superior temporal cortex has also been implicated in automatic, motor timing network (Lewis and Miall, 2003b) though more anteriorly than the region we observed. More consistent with our localization, posterior regions have been activated during duration discrimination (Ferrandez et al., 2003; Coull et al., 2004). Recently, right posterior superior temporal cortex and TPJ have been hypothesized to serve as part of a "when pathway" (Battelli et al., 2007). Though, evidence of laterality remains inconclusive, recent fMRI studies implicate left TPJ (Davis et al., 2009) and several patient studies selected for right hemisphere damage and therefore cannot make claims about the role of the left hemisphere. In this context, the left posterior superior temporal cortex may be involved with shared processing 
of spatio-temporal estimations that are used for both motor planning and perceptual estimations - perhaps integrating the dorsal and ventral streams.

Right posterior parietal cortex has been widely implicated in size estimations (Milner and Harvey, 1995; Milner et al., 1998; Ferber and Karnath, 2001) and more recently in processing in temporal order (Battelli et al., 2001; Snyder and Chatterjee, 2004; Becchio and Bertone, 2006; Correa et al., 2006). In our sample, damage to the white matter undercutting the left supramarginal gyrus and damage to right angular gyrus were correlated with impaired performance across both domains. The presence of partially overlapping size and TOJ representation in posterior parietal cortex is consistent with interaction between processing streams; the functional nature of how these systems overlap remains undefined.

\section{INDEPENDENT CORTICAL REPRESENTATIONS}

Voxel lesion symptom mapping performed on the residuals of TOJ regressed on size judgment and size judgment regressed on TOJ reveal regions that are vulnerable to impairment in each domain that cannot be accounted for by vulnerabilities in the other. Left frontal operculum damage impaired TOJ judgments not accounted for by size judgment deficits. Previously, right frontal operculum has been found to be involved with temporal decision-making (Rao et al., 2001) while left frontal operculum has been involved with temporal cuing (Coull and Nobre, 1998) and perception of temporal rhythmic patterns (Schubotz et al., 2000; Schubotz and von Cramon, 2001). Therefore, this region of left frontal operculum may mediate attentional mechanisms directed specifically at temporal segments.

Right posterior superior temporal cortex damage impaired size judgments that could not be accounted for by TOJ judgment deficits. Therefore, while left superior temporal cortex seems to be involved in shared processing of size and temporal order as described above, right superior temporal cortex appears independently engaged with size judgments.

Left posterior medial temporal, supramarginal gyrus and left angular gyrus damage impaired TOJ performance but not size judgment performance. Recently, a theoretical distinction between explicit timing and temporal expectation has been proposed (Coull and Nobre, 2008). Temporal expectation tasks such as temporal cuing or serial prediction tasks that implicitly call upon temporal information preferentially activate left premotor and left inferior parietal cortices. Though temporal sequencing and simultaneity

\section{REFERENCES}

Amorapanth, P. X., Widick, P., and Chatterjee,A. (2009). The neural basis for spatial relations. J. Cogn. Neurosci. 8,1739-1753.

Bates, E., Wilson, S. M., Saygin, A. P., Dick, F., Sereno, M. I., Knight, R. T., and Dronkers, N. F. (2003). Voxelbased lesion-symptom mapping. Nat. Neurosci. 6, 448-450.

Battelli, L., Cavanagh, P., Intriligator, J., Tramo, M. J., Henaff, M. A., Michel, F., and Barton, J. J. (2001). Unilateral right parietal damage leads to bilateral

studies have not yet been included in reviews of implicit timing tasks, it seems reasonable that they are dependent on similar cortical structures, which we observe in our findings.

We found spatially and functionally distinct subregions of the right supramarginal gyrus survived the residual analysis for both domains. Specifically, the bulk of the size related region was found to be more inferior than the bulk of the TOJ related regions, though the size region does extend to more superior supramarginal gyrus. Taken together, we conclude that within posterior parietal cortex, time judgments are represented bilaterally, although more uniquely on the left, and processing unique to size judgments are right lateralized. One hypothesis for bilateral involvement during TOJ is that the left and right superior temporal/posterior parietal regions operate as comparators (Davis et al., 2009) though this has not been tested. The nature of the spatial and functional independence of size judgment and TOJ processing within right posterior parietal cortex remains undefined.

\section{FINAL IMPLICATIONS FOR AN INTERNAL CLOCK}

Internal clock models are one class of models that describe how units of time might be represented. The putative clock stage of these models purports a pacemaker that generates a pulse that maps lawfully onto the passage of time. These pulses are integrated by an accumulator which transforms the pulses to a representation that can be manipulated or stored in memory (Gibbon, 1977). The TOJ data provides insight to the neural mechanisms underlying the clock stage as this task could be viewed as assessing the limits of temporal resolution (Correa et al., 2006). We reason that impaired performance on the TOJ tasks reflects an increase in the just noticeable difference of temporal durations which might map onto a widening of the pulse-width input into a neural accumulator or slow the "refresh rate" needed for temporal segregation across discrete snapshots. Therefore, regions we found to be uniquely vulnerable to insensitivity to temporal onset differences, including left frontal operculum, left precentral gyrus, left angular gyrus and bilateral supramarginal gyrus support subprocesses of the clock stage such as generation of the pulses from the pacemaker or entry into the accumulator.

\section{ACKNOWLEDGMENTS}

The authors wish to acknowledge Marianna Stark for coordinating the patient database and Bianca Bromberger for assistance in creating figures.

studies. Curr. Opin. Neurobiol. 18, 120-126.

deficit for high-level motion. Neuron 32, 985-995.

Battelli, L., Cavanagh, P., Martini, P., and Barton, J. J. (2003). Bilateral deficits of transient visual attention in right parietal patients. Brain 126, 2164-2174.

Battelli, L., Pascual-Leone, A., and Cavanagh, P. (2007). The "when" pathway of the right parietal lobe. Trends Cogn. Sci. 11, 204-210.

Battelli, L., Walsh, V., Pascual-Leone, A., and Cavanagh, P. (2008). The "when" parietal pathway explored by lesion
Becchio, C., and Bertone, C. (2006). Time and neglect: abnormal temporal dynamics in unilateral spatial neglect. Neuropsychologia 44, 2775-2782.

Burr, D., and Morrone, C. (2006). Time perception: space-time in the brain. Curr. Biol. 16, R171-R173.

Casasanto, D., and Boroditsky, L. (2008). Time in the mind: using space to think about time. Cognition 106, 579-593.
Cavina-Pratesi, C., Goodale, M. A., and Culham, J. C. (2007). FMRI reveals a dissociation between grasping and perceiving the size of real $3 \mathrm{D}$ objects. PLoS ONE 2, e424. doi:10.1371/journal.pone.0000424.

Chatterjee, A. (2005). A madness to the methods in cognitive neuroscience? J. Cogn. Neurosci. 17, 847-849.

Corballis, M. C. (2009). Comparing a single case with a control sample: correction and further comment. Neuropsychologia 47, 2696-2697. 
Correa,A., Sanabria, D., Spence, C., Tudela, P., and Lupianez, J. (2006). Selective temporal attention enhances the temporal resolution of visual perception: evidence from a temporal order judgment task. Brain Res. 1070, 202-205.

Coull, J., and Nobre, A. (2008). Dissociating explicit timing from temporal expectation with $\mathrm{fMRI}$. Curr. Opin. Neurobiol. 18, 137-144.

Coull, J. T. (2004). fMRI studies of temporal attention: allocating attention within, or towards, time. Brain Res. Cogn. Brain Res. 21, 216-226.

Coull, J. T., Nazarian, B., and Vidal, F. (2008). Timing, storage, and comparison of stimulus duration engage discrete anatomical components of a perceptual timing network. J. Cogn. Neurosci. 20, 2185-2197.

Coull, J. T., and Nobre, A. C. (1998). Where and when to pay attention: the neural systems for directing attention to spatial locations and to time intervals as revealed by both PET and AMRI. J. Neurosci. 18, 7426-7435.

Coull, J. T., Vidal, F., Nazarian, B., and Macar, F. (2004). Functional anatomy of the attentional modulation of time estimation. Science 303, 1506-1508.

Crawford, J. R., Garthwaite, P. H., and Howell, D. C. (2009). On comparing a single case with a control sample: an alternative perspective. Neuropsychologia 47, 2690-2695.

Critchley, M. (1953). The Parietal Lobes. London: Edward Arnold.

Danckert, J., Ferber, S., Pun, C., Broderick, C., Striemer, C., Rock, S., and Stewart, D. (2007). Neglected time: impaired temporal perception of multisecond intervals in unilateral neglect. J. Cogn. Neurosci. 19, 1706-1720.

Davis, B., Christie, J., and Rorden, C. (2009). Temporal order judgments activate temporal parietal junction. J. Neurosci. 29, 3182-3188.

DeLong, A. J. (1981). Phenomenological space-time: toward an experiential relativity. Science 213, 681-683.

Devinsky, O., and D'Esposito, M. (2004). Neurology of Cognitive and Behavioral Disorders. Oxford: NY: Oxford University Press.

Faillenot, I., Toni, I., Decety, J., Gregoire, M., and Jeannerod, M. (1997). Visual pathways for object-oriented action and object recognition: functional anatomy with PET. Cereb. Cortex 7, 77-85.

Farah, M. J., and Wolpe, P. R. (2004). Monitoring and manipulating brain function: new neuroscience technologies and their ethical implications. Hastings Cent. Rep. 34, 35-45.

Fellows, L. K., Heberlein, A. S., Morales, D. A., Shivde, G., Waller, S., and Wu, D. H. (2005). Method matters: an empirical study of impact in cognitive neuroscience. J. Cogn. Neurosci. 17, 850-858.

Ferber, S., and Karnath, H.-O. (2001). Size perception in hemianopia and neglect. Brain 124, 527-536.

Ferrandez, A. M., Hugueville, L., Lehericy, S., Poline, J. B., Marsault, C., and Pouthas, V. (2003). Basal ganglia and supplementary motor area subtend duration perception: an fMRI study. Neuroimage 19, 1532-1544.

Fraisse, P. (1963). The Psychology of Time. Westport, CT: Greenwood Press.

Gibbon, J. (1977). Scalar expectancy theory and Weber's Law in animal timing. Psychol. Rev. 84, 279-325.

Goodale, M. A., and Milner, A. D. (1992). Separate visual pathways for perception and action. Trends Neurosci. 15, 20-25.

Goodale, M. A., Milner, A. D., Jakobson, L., and Carey, D. (1991). A neurological dissociation between perceiving objects and grasping them. Nature 349, 154-156.

Husain, M., and Rorden, C. (2003). Nonspatially lateralized mechanisms in hemispatial neglect. Nat. Rev. Neurosci. 4, 26-36.

Husain, M., Shapiro, K., Martin, J., and Kennard, C. (1997). Abnormal temporal dynamics of visual attention in spatial neglect patients. Nature 385 , 154-156.

Johnston, A., Arnold, D. H., and Nishida, S. (2006). Spatially localized distortions of event time. Curr. Biol. 16, 472-479.

Kant, I. (1929). Kritic der Reinen Vernunft. Trans. Critique of Pure Reason, Norman Kemp Smith. New York: St. Martin's Press.

Kimberg,D.Y., Coslett,H.B., and Schwartz, M. F. (2007). Power in voxel-based lesion-symptom mapping. J. Cogn. Neurosci. 19, 1067-1080.

Leon, M. I., and Shadlen, M. N. (2003). Representation of time by neurons in the posterior parietal cortex of the macaque. Neuron 38, 317-327.

Lewis, P. A., and Miall, R. C. (2003a). Brain activation patterns during measurement of sub- and suprasecond intervals. Neuropsychologia 41, 1583-1592.

Lewis, P. A., and Miall, R. C. (2003b). Distinct systems for automatic and cognitively controlled time meas- urement: evidence from neuroimaging. Curr. Opin. Neurobiol. 13, 250-255.

Milner, A. D., and Harvey, M. (1995). Distortion of size perception in visuospatial neglect. Curr. Biol. 5, 85-89.

Milner, A. D., Harvey, M., and Pritchard, C. L. (1998). Visual size processing in spatial neglect. Exp. Brain Res. 123, 192-200.

Mishkin, M., and Ungerleider,L. G. (1983). Object vision and spatial vision: two cortical pathways. Trends Neurosci. 6, 414-417.

Mitchell, C. T., and Davis, R. (1987). The perception of time in scale model environments. Perception 16, 5-16.

Morrone, M. C., Ross, J., and Burr, D. (2005). Saccadic eye movements cause compression of time as well as space. Nat. Neurosci. 8, 950-954.

Nichols, T. E., and Holmes, A. P. (2002). Nonparametric permutation tests for functional neuroimaging: a primer with examples. Hum. Brain Mapp. 15, 1-25.

Nobre, A. C., Correa, A., and Coull, J. (2007). The Hazards of Time. Curr. Opin. Neurobiol.17, 465-70.

Onoe, H., Komori, M., Onoe, K., Takechi, H., Tsukada, H., and Watanabe, Y. (2001).Cortical networks recruited for time perception: a monkey positron emission tomography (PET) study. Neuroimage 13, 37-45.

Rao, S. M., Mayer, A. R., and Harrington, D. L. (2001). The evolution of brain activation during temporal processing. Nat. Neurosci. 4, 317-323.

Robertson, I. H., Mattingley, J. B., Rorden, C., and Driver, J. (1998). Phasic alerting of neglect patients overcomes their spatial deficit in visual awareness. Nature 395, 169-172.

Rorden, C., and Brett, M. (2000). Stereotaxic display of brain lesions. Behav. Neurol. 12, 191-200.

Rorden, C., and Karnath, H. O. (2004). Using human brain lesions to infer function: a relic from a past era in the fMRI age? Nat. Rev. Neurosci. 5, 813-819.

Rorden, C., Mattingley, J. B., Karnath, H.-O., and Driver, J. (1997). Visual extinction and prior entry: impaired perception of temporal order with intact motion perception after unilateral parietal damage. Neuropsychologia 35, 421-433.

Schubotz, R. I., Friederici, A. D., and von Cramon,D.Y.(2000). Time perception and motor timing: a common cortical and subcortical basis revealed by fMRI. Neuroimage 11, 1-12.
Schubotz, R. I., and von Cramon, D. Y. (2001). Functional organization of the lateral premotor cortex: fMRI reveals different regions activated by anticipation of object properties, location and speed. Brain Res. Cogn. Brain Res. 11, 97-112.

Shapiro, K., Hillstrom, A. P., and Husain, M. (2002). Control of visuotemporal attention by inferior parietal and superior temporal cortex. Curr. Biol. 12, 1320-1325.

Snyder, J. J., and Chatterjee, A. (2004). Spatial-temporal anisometries following right parietal damage. Neuropsychologia 42, 1703-1708.

Treisman, M. (1963). Temporal discrimination and the indifference interval. Implications for a model of the "internal clock”. Psychol. Monogr. 77, 1-31.

Walsh, V. (2003). A theory of magnitude: common cortical metrics of time, space and quantity. Trends Cogn. Sci. 7, 483-488.

Wencil, E. B., Coslett, H. B., Aguirre, G. K., and Chatterjee, A. (2010). Carving the clock at its component joints: neural bases for interval timing. $J$. Neurophysiol. jn.00029.02009.

Woo, S.-H., Kim, K.-H., and Lee, K.-M. (2009). The role of the right posterior parietal cortex in temporal order judgment. Brain Cogn. 69, 337-343.

Wu, D. H., Waller, S., and Chatterjee, A. (2007). The functional neuroanatomy of thematic role and locative relational knowledge. J. Cogn. Neurosci. 19, 1542-1555.

Xuan, B., Zhang, D., He, S., and Chen, X. (2007). Larger stimuli are judged to last longer. J. Vis. 7, 1-5.

Conflict of Interest Statement: The authors declare that the research was conducted in the absence of any commercial or financial relationships that could be construed as a potential conflict of interest.

Received: 26 April 2010; paper pending published: 20 May 2010; accepted: 08 August 2010; published online: 14 September 2010.

Citation: Wencil EB, Radoeva $P$ and Chatterjee A (2010) Size isn't all that matters: noticing differences in size and temporalorder. Front. Hum. Neurosci. 4:171. doi: 10.3389/fnhum.2010.00171

Copyright (C) 2010 Wencil, Radoeva and Chatterjee. This is an open-access article subject to an exclusive license agreement between the authors and the Frontiers Research Foundation, which permits unrestricted use, distribution, and reproduction in any medium, provided the original authors and source are credited. 